\title{
CONTAMINATION OF SOILS BY HEAVY METALS AND THEIR INFLUENCE ON THE ENVIRONMENT IN BULGARIA \\ Tsvetelina Petrova ${ }^{1}$, Iskra Simova ${ }^{2}$, Martin Pushkarov ${ }^{3}$, Rositsa Velichkova ${ }^{4}$, Detelin Markov ${ }^{5}$
}

\begin{abstract}
Heavy metals are one of the major pollutants. The main sources of pollution are industry, transport, the use of pesticides and mineral fertilizers in agriculture, the military industry, etc.

Pollution is mostly around businesses, highways and treated terrains and they are polymetallic, mostly from $\mathrm{Cu}, \mathrm{Pb}, \mathrm{Zn}, \mathrm{Cd}$, $\mathrm{Hg}$, As and others.

In the current paper the main heavy metals for Bulgaria for the different sources of pollution are presented. An analysis of data for problem areas is made, according to sources of pollution. An analysis of the impact of pollutants on the environment is also conducted.
\end{abstract}

UDC Classification: 5(502), DOI: 10.12955/cbup.v7.1488

Keywords: soil, heavy metal, environment, pollution

\section{Introduction}

Universal pollution of the environment, air and water naturally reflects on a soil contamination. Soils pollutants include liquid and solid pollutants from households however, industry and agriculture are the major polluters. The main soil pollutants are metals and their compounds used in agriculture, fertilizers of different origins, pesticides, radioactive substances, etc.

The presence of pollutants which exceeded limit values (LV) can lead to negative impacts towards the production of food.

Heavy metal soil pollution has a direct social significance, especially for children. Playing outdoors, small children could take many contaminants orally, not only from contaminated soils, but also from the food and the air.

There is a group of metals for which the term "heavy" has meaning of "toxic". In recent decades, the term "heavy metals" has appeared in specialized scientific literature, which is associated with a negative impact and the perception of something dangerous and toxic to living organisms (animals and plants). Heavy metals include all metals with a relative atomic mass greater than 40 or with a density greater than $5 \mathrm{~g} / \mathrm{cm}^{3}$. At the same time, some of them: $\mathrm{Cu}, \mathrm{Zn}, \mathrm{Mo}, \mathrm{Co}, \mathrm{Mn}, \mathrm{Fe}$, etc. play an important role in plants and animals (Dinev et al., 2011; Pollution sources, 2007)

The main sources of heavy metal pollution are industry, transport, the use of pesticides and mineral fertilizers in agriculture, the military industry, etc.

This work summarizes the characteristic pollutants from different origins in Bulgaria. An analysis of data for problematic areas is proposed, according to the sources of pollution/contamination. This is also scrutinized by an analysis of the impact of pollutants on the environment.

\section{Assessment of soil contamination}

Within the last 50-60 years, as a result of the rapid development of scientific and technological progress, inadequate management or criminal negligence, a number of industrial breakdowns and accidents occurred in large industrial sites and nuclear power plants. In many parts of the world, pollution covers almost $100 \%$ of the water bodies, nitrogen oxides are 10-15 times above the LV, sulfur anhydride almost 50-60 times above the LV, and almost half of the population live in a polluted

\footnotetext{
${ }^{1}$ College of Energy and Electronics (CEE), Department of Energy and Mechanical Engineering, Sofia, Bulgaria, tzvetelina.petrova@tu-sofia.bg

${ }^{2}$ Technical University of Sofia, Department Hydroaerodynmics and hydraulic machines, Sofia, Bulgaria, iskrasimova@gmail.com

${ }^{3}$ Technical University of Sofia, Department Hydroaerodynmics and hydraulic machines, Sofia, Bulgaria, martotoo@abv.bg

${ }^{4}$ Technical University of Sofia, Department Hydroaerodymaics and hydraulic machines, Sofia, Bulgaria, rositsavelichkova@abv.bg

${ }^{5}$ Technical University of Sofia, Department Hydroaerodymaics and hydraulic machines, Sofia, Bulgaria, detmar@abv.bg
} 
atmosphere, and are exposed to polluted food and water (Dinev et al., 2011). Salinization and pollution of arable land, destruction of forests, changing of the atmosphere, water scarcity and pollution, etc. have caused more than 400 million people worldwide to live in polluted ecological conditions and more than 230 million in a water deficit (Dinev et al., 2011). Therefore, the issues of biosphere degradation and pollution of its components are a new element of ecological and human security and are a problem for the survival of mankind.

Soil pollution is not a new problem, but its nature has begun to be discussed only after the Environmental Conference held in Stockholm in 1972. Soil pollution is considered in when the concentration of pollutants is bigger than the LV. It is also known that mankind uses about 500,000 chemical compounds and substances, and more than 100,000 are man-made, e.g. are not found in nature, of which 15,000 trigger anxiety, and 500 substances entering the soil substrate are considered dangerous and harmful to living creatures and threaten the fertility of soil and human health.

Soil pollution with heavy metals such as cadmium, lead, chromium, copper, zinc, mercury and arsenic are worrying. Heavy metals are naturally present in soils, but their increased concentration is due to industry, agriculture, waste incineration, fuel burning, road transport, etc.

The pollution of agricultural lands with heavy metals leads to a reduction in yields and an increase in the amount of harmful elements in plant production, and hence to a shift in the food chain. The accumulation of heavy metals in meadows occurs mainly in the surface five centimeters and during grazing goes directly into the animals.

Heavy metals are toxic and obstruct soil micro-organisms' activity. Heavy metal concentration in soils can last for decades (Vasilev et al., 2011).

The main source of pollution is the large industrial plants for ferrous and non-ferrous metals and ore extraction activities, which are a source of pollution through wastewater and tailings ponds for the disposal of waste from primary processing. Soil pollution is the result of their uncooperative exploitation, insufficient and inefficient water treatment facilities and low ecological control.

When assessing soil contamination according to the Bulgarian State Standard, the major groups of pollutants which change the acid-alkali and oxidation conditions are defined. As a consequence, they deteriorate the quality of the soil. Based on the impact and stability of the soils on the substances they are classified as:

1. highly resistant - clayey, carbonate, saturated with bases and rich in organic soils;

2. medium resistant - sandy clayey, neutral medium saturated with bases and rich in humus soils;

3. poorly resistant - sandy and unsaturated with base soils.

By the contamination level the soils are divided into:

1. highly contaminated - the content of the elements exceeds several times the LV, which results in deterioration of the soil properties;

2. moderately contaminated - the content of the elements in the soil exceeds the LV, but it does not lead to a change in the soil properties;

3. slightly polluted - the content of the elements is between the background and LV (Green book, 2003; Water, 2007).

\section{Analysis of the Problematic Areas in Bulgaria}

In this part of the paper, an analysis of the problem areas in Bulgaria is made. Results for LV are given and an assessment of contaminant based on Ordinance No. 3 on the content of harmful substances in soils (SG, 1979 , SG,1997) is made, using a coefficient of technogenicity Ct (ratio between the measured content and the relevant LV).

The total area of the polluted lands above the maximum concentration limits amounts to 43660 ha, which represents $0.7 \%$ of the agricultural territories of the country. $61.3 \%$ of the polluted soils are close to industrial enterprises, of which 8160 ha are polluted five times above the LV. The areas in the three-four kilometer zones around the major industrial sites (Eliseyna, Kardzhali, Pirdop, Plovdiv, Kremikovtzi, etc.) are the most affected. They are mapped in detail (in M 1: 5000 and M 1: 10 000) and the ecologically polluted agricultural lands from industrial activity have been investigated. All 
studies exist as digital models and can be used to impose different land use regimes depending on the degree of pollution (Vasilev et al., 2011).

The problematic areas in Bulgaria according the contaminants are shown on Figure 1

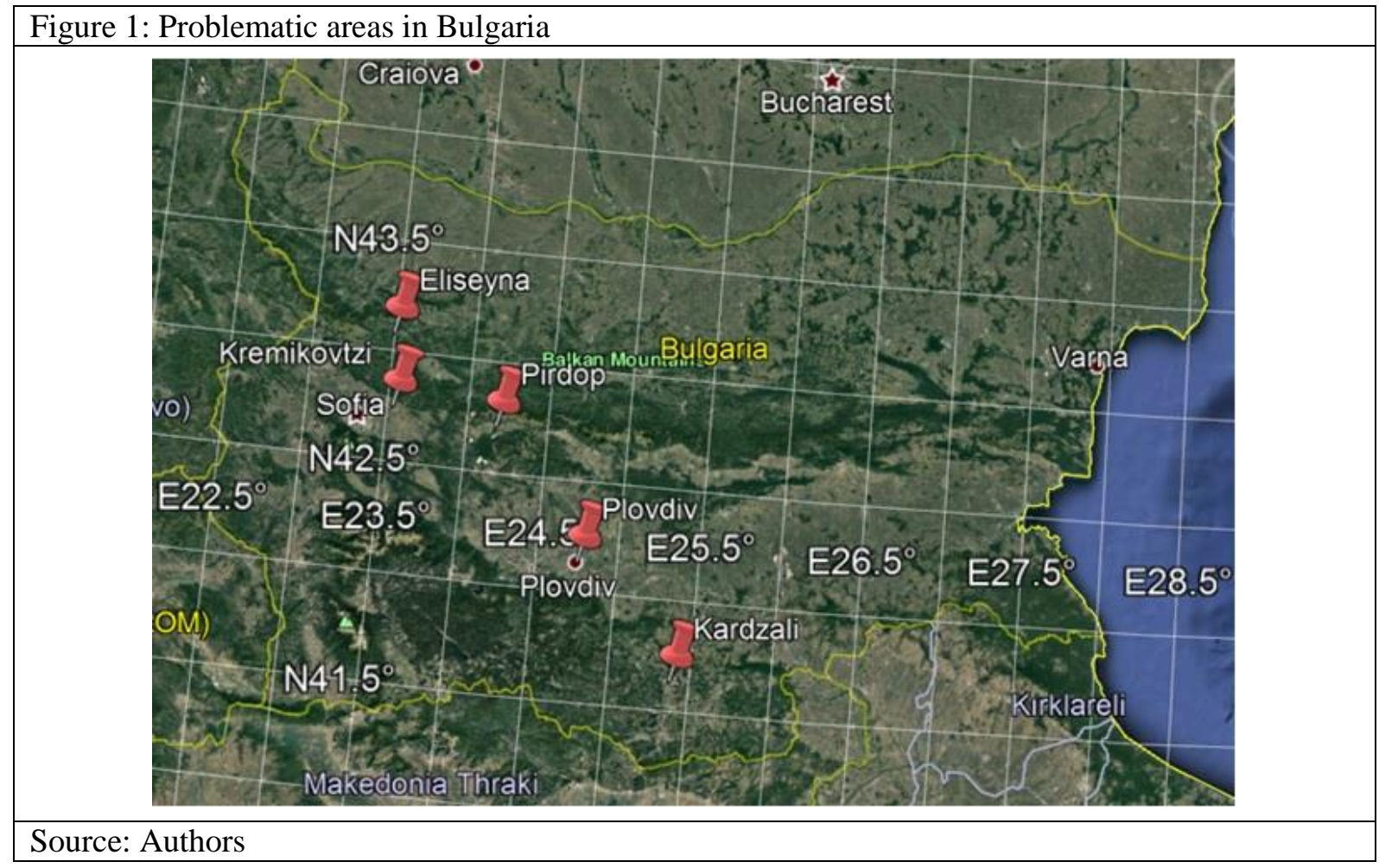

The following figures (Figures 1 - 6) show the pollution in problem areas in Bulgaria, depending on the concentration of LV.

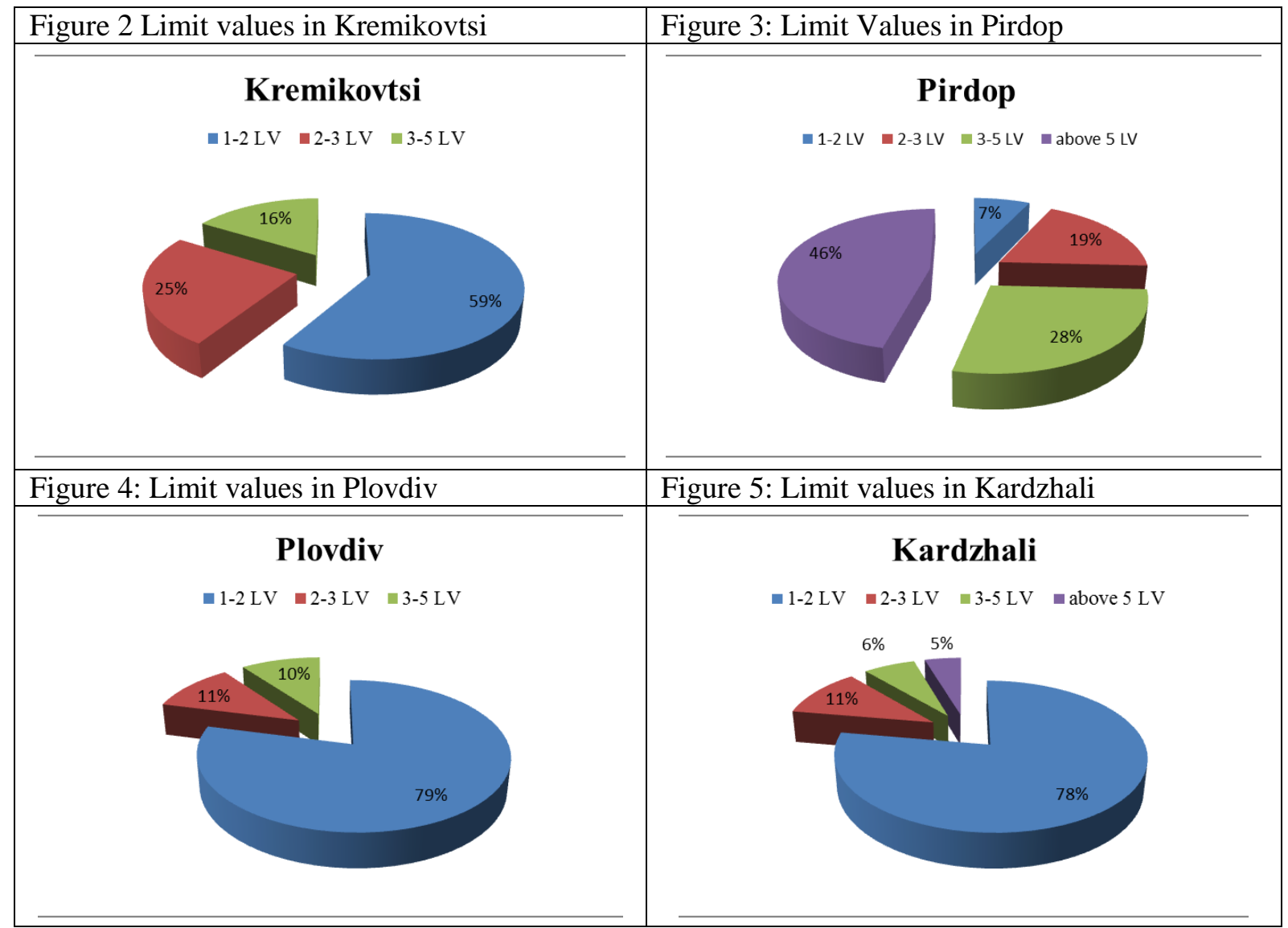




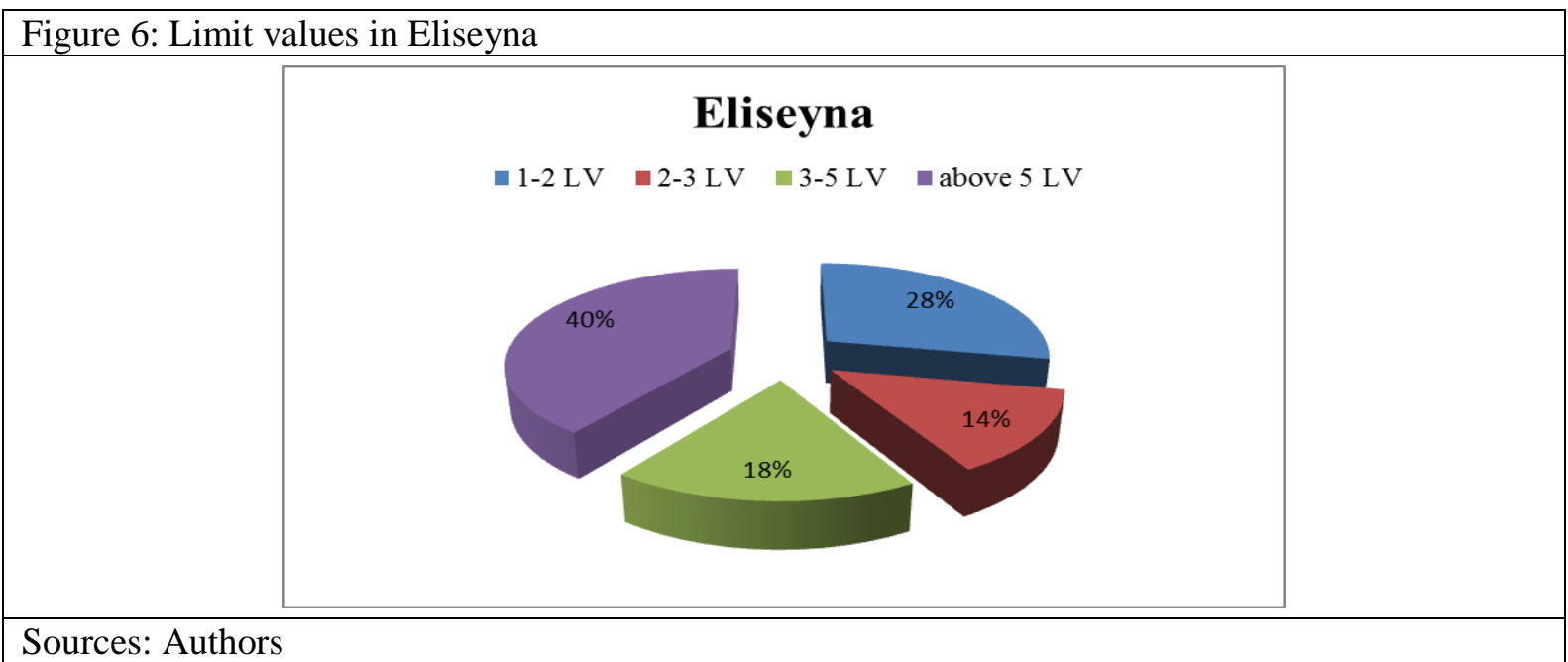

These results present the problematic areas that overrun the LV. In Kremikovtsi, Plovdiv and Kardhzali the most excess is between 1-2LV, while in Pirdop and Eliseyna it is above $5 \mathrm{LV}$.

Sampling and analysis are performed in accordance with ISO Standards No. 11466 and 11047 in the basic RIEW laboratories and the accredited laboratory of the EEA.

The National Network for Monitoring and Control of Soil Pollution with Heavy Metals and Metals is organized according to the source of pollution. The number of points in the monitoring and control network is 303 , broken down by sources of pollution as follows: industry (92) chemistry (80 pieces), irrigation (52 pieces), road transport (79 pieces)

The total number of checkpoints included in the national monitoring network is 92 . They are located both in environmentally-congested areas and close to most industrial areas of larger cities. Subject to control are also the areas with mining activity, despite the cessation of activity in most of them. Depending on the controlled potential sources, the results obtained are as follows:

"Kremikovtzi" AD - Sofia: 7 control points, with no significant change in the accumulated quantity of heavy metals in recent years. Yana, 500 meters from the plant, maintains levels of $3.5 \mathrm{Kt}$ for lead up to $4.5 \mathrm{Kt}$ for As. The modified land use of corn in cereal crops (barley) is appropriate for these overbearing contents (Vasilev et al., 2011).

Miner Pirdop Med AD: 5 control points. The accumulation of additional amounts of heavy metals, predominantly $\mathrm{Cu}$, as well as the dynamics of soil acidity, is recorded.

The soil and land use in the area, combined with the abundant copper, arsenic and $\mathrm{SO}_{2}$ oxide content, make the area problematic with a view to implementing a policy for sustainable land management and land use.

KCM AD - Plovdiv: 10 control points without significant change in pollution. At KCM station located $500 \mathrm{~m}$ southeast of the plant, the measured contents are 26.4, 6.8 and $13.9 \mathrm{Kt}$, respectively, for lead, zinc and cadmium. The soils in the area of pollution are carbonates, which block the digestibility of heavy metals by the vegetation. Traditional land use in the area of vineyards and orchards (accumulating in the heavy metals) also favors countering the negative impact of existing pollution.

"OCC" AD - Kardzhali: 7 checkpoints, registered change in the contents of lead, zinc and cadmium at 2 points, located up to $1 \mathrm{~km}$ from the emitter (Vasilev et al., 2011).

"MK" - Eliseina: 5 control points, 4 of them located in yard and 1 in levels. Extremely high levels of heavy metals in the soils. There is a real health risk due to land use for agricultural purposes.

In Figures 7-11, the contaminant in problem areas in Bulgaria according $\mathrm{Ct}$ coefficient and the number of the control points mention above are summarized.

\section{Conclusion}

Soils are subject to ever stronger pressures created or reinforced by human activities such as inappropriate agricultural and forestry practices, industrial activities, landfilling, tourism or city planning. These activities damage the ability of soils to continue to fulfill their essential functions. In 
addition, soil degradation has a strong impact on other environmental components, such as water and biodiversity, as well as on climate change, human health and food safety.
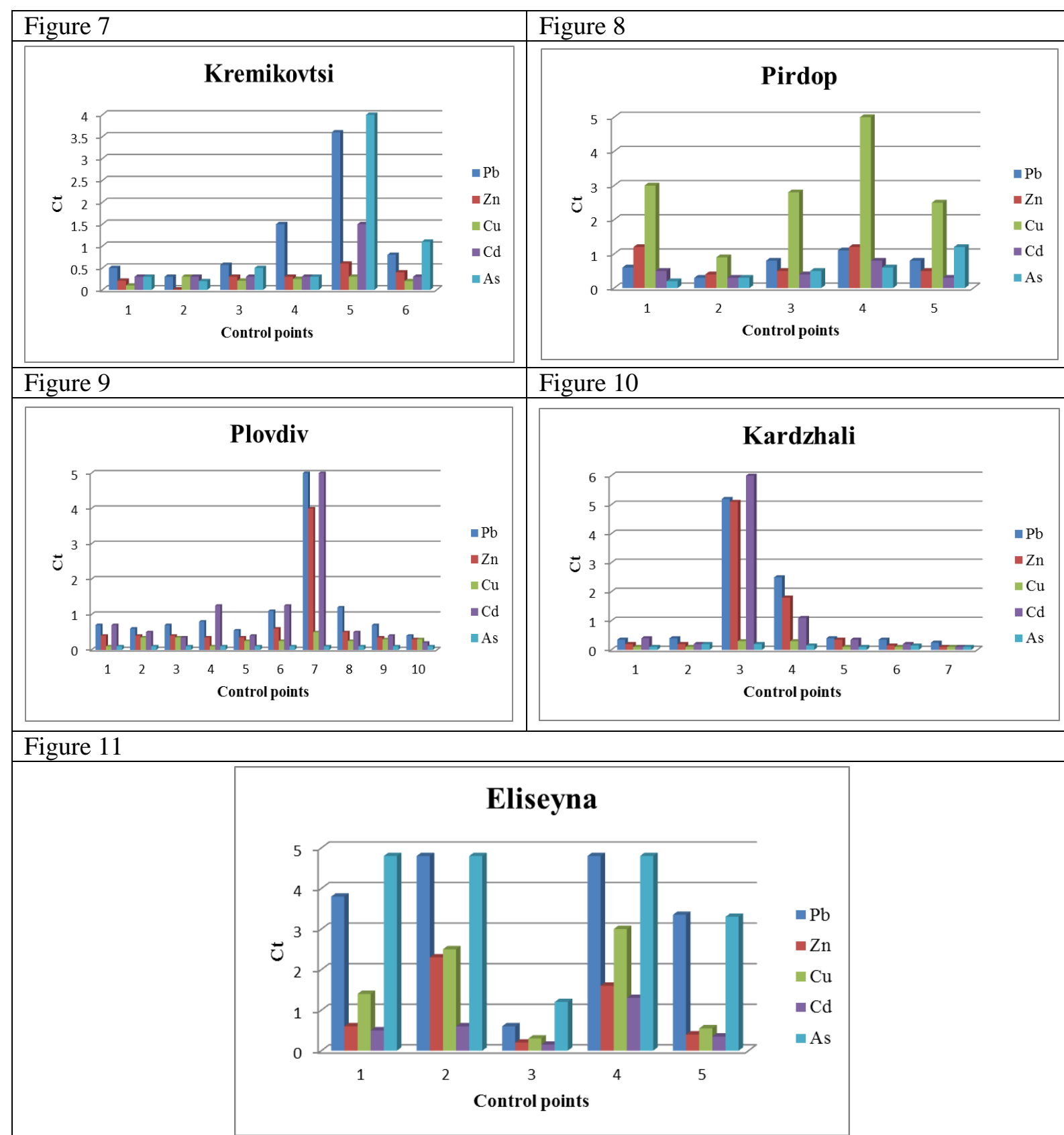

Sources: Authors

Strategic approaches to soil contamination require both preventive measures and remediation of contaminated soils and groundwater. Preventive measures consist of reducing industrial pollution and soil and groundwater protection.

The presented result can be helpful for more detailed assessment of the soil contamination and preventive measures in the future.

\section{Acknowledgement}

This work has been carried out in project No BG05M2OP001-2.009-0033 "Promotion of Contemporary Research Through Creation of Scientific and Innovative Environment to Encourage Young Researchers in Technical University - Sofia and The National Railway Infrastructure Company in The Field of Engineering Science and Technology Development" within the Intelligent Growth Science and Education Operational Programme co-funded by the European Structural and Investment Funds of the European Union and under contract N 192ПД0024-02 Scientific and research centre at 
Technical university of Sofia for Assistance of PhD Student, 2019-2020 " Development of a specialized test-rig for the experimental study of acid mining waters"

\section{References}

Dinev, N., E. Filcheva. (2011). Soil monitoring: indicators of heavy metal contamination and application of GPS techniques in risk assessment. Soil Science, Agrochemistry and Ecology, Year XLV, Appendix 1-4, pp86-92.

Green book. (2003). Retrieved from http://eea.government.bg/eea/bg/publicat/2003/quality/soil/soil1.htm

Pollution sources: Mining. (2007). Retrieved from http://www.pollutionissues.com/Li-Na/Mining.html\#ixzz5hn6sK5Kr

State gaztete $36 / 79,54 / 97$.

Vasilev, A., N. Dinev. (2011). Ecotoxicological assessment of soils contaminated with heavy metals in the area of Kremikovtzi and KCM - Plovdiv. Soil Science, Agrochemistry and Ecology, Year XLV, Appendix 1-4, 72-76.

Water. (2017). Retrieved from https://www.moew.government.bg/bg/vodi/ 